\title{
EPTCAST - UNA PROPUESTA PARA LA DIVULGACIÓN CIENTÍFICA EN LA EDUCACIÓN PROFESIONAL Y TECNOLÓGICA BRASILEÑA
}

\section{ARTÍCULO ORIGINAL}

RAMOS, Rogério Luiz da Silva ${ }^{1}$, FECURY, Amanda Alves², OLIVEIRA, Euzébio de ${ }^{3}$, DENDASCK, Carla Viana ${ }^{4}$, DIAS, Claudio Alberto Gellis de Mattos $^{5}$

RAMOS, Rogério Luiz da Silva. Et al. EPTCast - Una propuesta para la divulgación científica en la educación profesional y tecnológica brasileña. Revista Científica Multidisciplinar Núcleo do Conhecimento. Año 06, Ed. 06, Vol. 07, págs. 89 y 103. Junio de 2021. ISSN: 2448-0959, Enlace de acceso: https://www.nucleodoconhecimento.com.br/educacion-es/profesional-y-tecnologica, DOI: 10.32749/nucleodoconhecimento.com.br/educacion-es/profesional-ytecnologica

\section{RESUMEN}

Este trabajo consiste en un informe de experiencia sobre el desarrollo de EPTCast, producto educativo desarrollado a lo largo del máster profesional en Formación Profesional y Tecnológica en Red Nacional (ProfEPT). Partiendo de la falta de divulgación científica específicamente enfocada en el campo de la Educación Profesional y Tecnológica Brasileña (EPT), este trabajo tiene como objetivo promover

\footnotetext{
${ }^{1}$ Licenciado en Comercio Exterior, Especialista en Ingeniería de Producción (UNINTER), Profesor e investigador del Instituto de Educación Básica, Técnica y Tecnológica de Amapá (IFAP), Estudiante del Programa de Posgrado en Educación Profesional y Tecnológica (PROFEPT IFAP).

${ }^{2}$ Biomédico, Doctor en Enfermedades Trópicas, Profesor e investigador del Curso Médico del Campus Macapá, Universidad Federal de Amapá (UNIFAP).

${ }^{3}$ Biólogo, Doctor en Enfermedades Trópicas, Profesor e investigador del Curso de Educación Física de la Universidad Federal de Pará (UFPA).

${ }^{4}$ Teóloga, Doctora en Psicoanálisis Clínica. Trabaja desde hace 15 años con Metodología Científica (Método de Investigación) en Orientación de Producción Científica para Estudiantes de Maestría y Doctorado. Especialista en Investigación de Mercados e Investigación en Salud, Estudiante de Doctorado en Comunicación y Semiótica (PUC SP).

${ }^{5}$ Biólogo, Doctor en Teoría e Investigación del Comportamiento, Profesor e investigador del Curso de Grado de Química del Instituto de Educación Básica, Técnica y Tecnológica de Amapá (IFAP) y del Programa de Posgrado en Educación Profesional y Tecnológica (PROFEPT IFAP).
} 
iniciativas en este sentido, contribuyendo así a la popularización de la cienticidad de esta área. Para ello, aquí se retrata el proceso de construcción y distribución vía podcast de un contenido de audio dirigido a la divulgación científica del EPT brasileño. En este sentido, inicialmente se aborda el tema de la brecha, y, por lo tanto, la necesidad de popularizar la cienticidad de esta área. En un segundo momento, se presenta el diseño y las tecnologías utilizadas en la construcción del objeto EPTCast. Popularizar la cienticidad de la Educación Profesional y Tecnológica brasileña es lo que se pretende a través de este trabajo - que no será el único. Por lo tanto, a través de lo expuesto en los apartados anteriores, se pretende contribuir a la popularización de una cienticidad que a veces aún no se entiende como tal.

Palabras clave: Podcast, Divulgación Científica, Educación Profesional y Tecnológica.

\section{INTRODUCCIÓN}

La divulgación científica es una práctica adoptada desde la premisa de la distancia entre la comunidad científica y el público no especializado. En este sentido, este esfuerzo comunicativo ha sido presentado como un instrumento discursivo pedagógico de construcción del conocimiento que tiene como objetivo acercar los procesos científicos al público en general (MOIRAND, et ali, 2016).

Por lo tanto, con el advenimiento de la difusión de la red mundial de computadoras a través de Internet, la divulgación científica encuentra más espacio para desarrollarse. Esto se percibe a través de la variedad de medios digitales donde se manifiesta esta actividad: desde textos en blogs, vídeosen plataformas dedicadas y también a través de podcasts (DE-LARA-GONZÁLEZ y DEL-CAMPO-CAÑIZARES, 2018).

Con su primera referencia fechada en 2004, el podcast de medios se ha vuelto popular en el campo de la comunicación. A partir de la creciente cantidad y variedad de opciones, la gente común, las empresas e incluso las compañías de medios tradicionales han estado produciendo y distribuyendo su contenido a través de este medio (RIVERA SALAS y MONTOYA MÁRQUEZ, 2019). 
Frente a lo ya consolidado, el medio podcast cuenta con procesos sencillos y accesibles, ya sea para su producción o consumo. Por lo tanto, esto se presenta como una razón para que este medio sea utilizado en la distribución de contenidos educativos. Sin embargo, cuando se trata de divulgación científica, este movimiento es menos frecuente (DANTAS-QUEIROZ, WENTZEL y QUEIROZ 2018).

Específicamente en lo que respecta a la divulgación científica en la Educación Profesional y Tecnológica Brasileña (EPT), hay un vacío. Entendida como un instrumento de ascensión social, esta área de investigación ha ido construyendo el conocimiento necesario para mejorar las condiciones de las clases trabajadoras populares para que, de hecho, la sociedad en su conjunto se desarrolle (MOURA, 2013).

\section{GOL}

Retratar el proceso de construcción del EPTCast con el fin de fomentar iniciativas más grandes y mejores, con el fin de contribuir a la popularización de la cienticidad producida en el campo del EPT brasileño.

\section{MATERIALES Y MÉTODOS}

Desarrollado por aquellos que ven la integración entre educación y trabajo como un camino para el desarrollo social de las clases trabajadoras populares, EPTCast se constituye a través de un contenido de audio dirigido a la divulgación científica del EPT brasileño. Distribuido a través del podcast de medios, se trata de un Producto Educativo iniciado en el primer semestre de 2020 y desarrollado a lo largo del máster profesional en Formación Profesional y Tecnológica en Red Nacional (ProfEPT).

El primer reto para la construcción de esta propuesta fue la creación de una identidad visual, que requirió el diseño de un nombre, un eslogan y un símbolo gráfico. Una identidad visual está, por regla general, diseñada para la mejor comunicación posible a través de signos verbales y/o no verbales (MILLER e TOMAN, 2014). Por lo tanto, dada la necesidad de distribución a través de Internet - que requiere fuertemente la 
presencia de recursos visuales para la propagación de contenidos (LIU, 2020) - esta fue la primera demanda en la construcción de este Producto Educativo.

Luego, para la concepción del nombre, inicialmente se dio preferencia por algo objetivo y que estaba directamente asociado con su modo de comunicación. En este sentido, y sobre todo, desde el entendimiento de que los nombres -ya sea para ideas, productos o servicios- siempre se construyen dentro de una estructura existente, lo que hace que un nuevo nombre lleve parte de otros (NDERITU PATRICK, 2016), optamos por aliar el acrónimo de 'Educación Profesional y Tecnológica' con la última parte del nombre de su medio dedistribución 'cast'. Como resultado, se creó el nombre EPTCast.

Luego, para la continuación del desarrollo de la identidad visual, se construyó un eslogan. Frase corta y rápida asociación con la idea propuesta por su nombre, un eslogan pretende presentar brevemente el concepto de obra (MILLER y TOMAN, 2014). Por lo tanto, optamos por describir los medios de distribución junto con el contenido propuesto. Pronto, el nombre EPTCast adoptó el siguiente lema: "Seu podcast sobre Educação Profissional e Tecnológica".

Finalmente, tras la concepción del nombre y su eslogan,fue seguido por la construcción de un símbolo gráfico. También conocido como logotipo, este es un elemento visual que tiene como objetivo la comunicación con el público interesado. En esta perspectiva, la identidad visual de una marca debe ser una herramienta de comunicación simple e inteligible (BOLHUIS et ali, 2015). Por lo tanto, elegimos el uso de un globo de diálogo. Finalmente, para la construcción de la elaborada idea, hicimos uso de la versión gratuita de la plataforma online para la creación de dibujos y layouts, Canva.com.

Tras el desarrollo de la identidad visual, la materialización del Producto Educativo EPTCast tuvo las actividades de producción y distribución. La producción consistió en el trabajo de scripting, captura y edición de contenido de audio. Para estas tareas, se utilizaron como instrumentos de trabajo el software de edición de texto Microsoft Word 
versión 2016, el software de edición de audio Audacity versión 2.3.1 y las funciones de audio de las plataformas YouTube (audiolibrary) y Freesound.org.

A su vez, la Distribución se realizó mediante el uso de la Anchor.fm. Para la identificación del material producido, los espacios dedicados a esta tarea se utilizaron directamente en la plataforma Anchor.fm, que insertó automáticamente los contenidos de EPTCast en las siguientes plataformas de audio digital: Apple Podcasts; Interruptor; Castbox; Google Podcasts; Nublado; Moldes de bolsillo; RadioPublic y Spotify.

\section{RESULTADOS Y DISCUSIONES}

El trabajo utilizado a lo largo del proceso de desarrollo de este Producto Educativo fue el resultado de horas de consumo previo de contenidos de divulgación científica distribuidos vía podcast. Sin embargo, debido a que existen raras referencias en el área del EPT brasileño en el caso de estas especificidades, los siguientes resultados pueden, al mismo tiempo, presentar limitaciones con respecto a su construcción y oportunidades para el desarrollo de propuestas más robustas.

\section{IDENTIDAD VISUAL}

A través del uso de la versión gratuita de la plataforma Canva.com (https:canva.com) se inició la construcción de la identidad visual del Producto Educativo EPTCast. Para su lienzo se eligió el tamaño de 1500 por 380 píxeles y el color \#000000. Para el nombre, la fuente tipográfica Montserrat Classic en estilo negrita con tamaño $50 \mathrm{y}$ color de la \#000000. Luego, se trabajóel slogan, que conservaba la misma fuente tipográfica y estilo aplicado al nombre, pero recibía cambios de tamaño y color, respectivamente 38 y \#fffff. Finalmente, se ha seleccionado un Globo de Diálogo dentro de la opción 'Formas' bajo 'Elementos' en la plataforma en uso. Después de la inserción, el elemento se redimensionó al tamaño 365 por 290 píxeles y el color \#ffbf00 (Figura 1). 
Figura 1 - Identidad visual del producto educativo EPTCast.

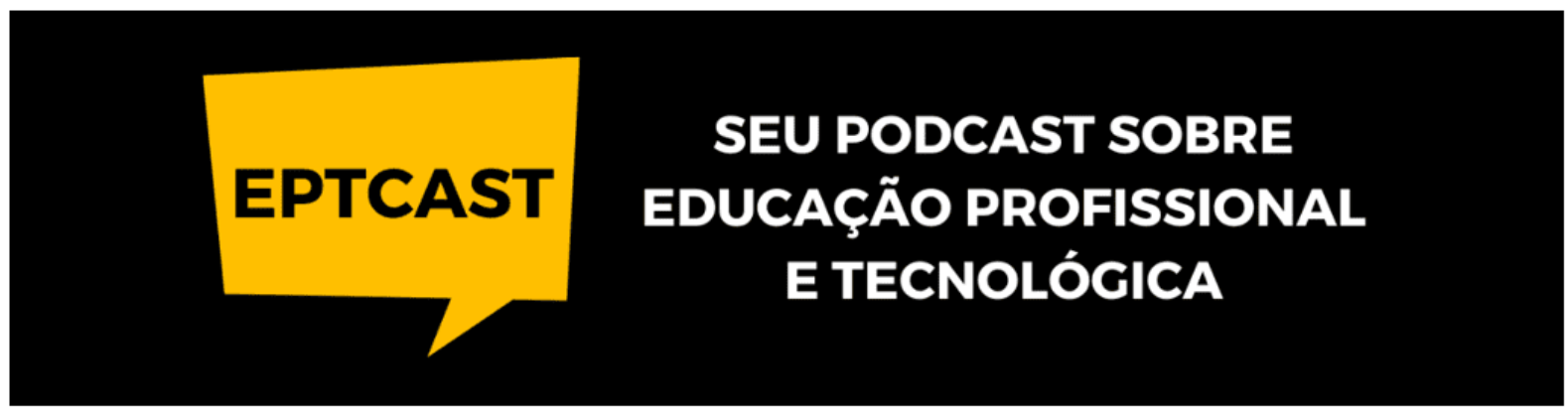

La identidad visual de este Producto Educativo fue diseñada para que pudiera ser aplicada en el futuro en espacios digitales y productos físicos promocionales. Por ello, desde el inicio de su construcción, se estableció que esta identidad debía caracterizarse a través de una imagen simple y de aplicabilidad múltiple.

Dentro de esta propuesta, al nombre, se aplicaron especificaciones que cumplían con lo previsto. Con el fin de mantener la característica central del nombre, el slogan conservaba la misma idea, sin embargo, dado el mayor número de letras, se redujo su tamaño y, para ser leído frente al lienzo, se cambió su color. Se aplicó el Globo de Diálogo para el reconocimiento de la propuesta comunicativa. Su color diferenciado y la opción de posicionar el nombre frente a él fue diseñado para que también pudieran presentarse de forma independiente al eslogan.

\section{PRODUCCIÓN}

De enero de 2020 a septiembre del mismo año, la producción de este Producto Educativo dio como resultado 19 audios (\#00 a \#18). Al principio - hasta la publicación del día 12 - el contenido fue desarrollado a través de temas recurrentes en la literatura del EPT brasileño. A partir del 14, comenzó a producir y publicar a través de sugerencias de temas enviados por la audiencia, que hizo uso de los canales de comunicación eptcast.com.br (comentarios), y el correo electrónico contato.eptcast.com.br. El audio 13 publicado tenía la intención de resumir el contenido presentado hasta ahora y estimular la participación de la audiencia. 


\section{SCRIPTING}

Después de la finalización de la identidad visual, el scripting se realizó mediante el uso del software propietario de edición de texto Microsoft Word, versión 2016 (https://office.com). A partir del uso de esta herramienta, se inició la construcción del contenido desarrollado en 03 partes, "Presentación", "Evolución" y "Conclusión" (Tabla 1).

La "Apresentação" se subdividió en otras 03 partes, donde, la primera 'Apertura', se dedicó a la bienvenida, presentación del locutor e identificación del programa. A continuación, 'Contexto', se centró en identificar la coyuntura del fenómeno abordado. Finalizando el bloque "Presentación", se utiliza el bloque 'Call to action',momento en el que se invita al público a interactuar por medios electrónicos al final del audio.

El siguiente momento, "Evolução", se dividió en 04 partes. El primero, 'Fenómeno', se dedicó al resumen sobre el objeto tratado. A continuación se realizaron 'Antecedentes' y 'Características' espacios centrados en el abordaje del proceso de desarrollo del fenómeno en cuestión y sus propiedades. Por último, 'Desdobramentos', se dedicó a las consecuencias en el contexto social, especialmente para educación y trabajo.

La "Conclusão" se dividió en 03 partes. La primera, 'Reflexão' se dedicó a observaciones sucintas sobre el tema tratado. El siguiente momento, 'Referencias', tuvo como objetivo informar al público sobre el lugar de alojamiento del material utilizado en la construcción del guion. Por último, se utilizó la función "Call to action". 
Tabla 1 - Esquema narrativo del guion.

\begin{tabular}{|c|c|c|}
\hline Apresentação & Evolução & Conclusão \\
\hline Abertura & Fenômeno & Reflexão \\
\hline (30 segundos) & (01 min. e 30 segs.) & (02 mins. e 30 segs.) \\
\hline Contexto & Antecedentes & Referências \\
\hline (01 minuto) & (01 min. e 30 segs.) & (30 segundos) \\
\hline \multirow{3}{*}{$\begin{array}{l}\text { Call to action } \\
(30 \text { segundos })\end{array}$} & Características & Call to action \\
\hline & (03 minutos.) & (01 minuto) \\
\hline & $\begin{array}{c}\text { Desdobramentos } \\
\text { (03 minutos.) }\end{array}$ & \\
\hline 02 minutos & 09 minutos & 04 minutos \\
\hline
\end{tabular}

\section{5 minutos}

Los elementos de composición del esquema narrativo fueron pensados de tal manera que el oyente comprendiera la relación entre el fenómeno tratado y la realidad actual en el contexto de la Educación y el Trabajo. El tiempo dedicado a cada división, y sus subdivisiones, se estipularon de tal manera que permitieran la estandarización, y por lo tanto una mejor organización para la construcción de futuros itinerarios.

Del total de 18 audios producidos, los números \#00, \#04, \#08, \#10 y \#12, debido a la naturaleza de sus propuestas, no cumplieron con el esquema anterior. $\mathrm{Y}$, a partir de la \#13, debido a la pluralidad entre las sugerencias de temas recibidos, el esquema narrativo sufrió algunos cambios en la sección "Evolución". Sin embargo, la esencia de lo que se construyó en el esquema presentado anteriormente, se conservó hasta el último tema publicado

En la Tabla 2 se muestra el número de audios y temas abordados. 
Tabla 2 - Temas dirigidos a cada audio.

\begin{tabular}{c|c}
\hline Número & Assunto \\
\hline$\# 00$ & O que é o EPTCast?"; \\
\hline$\# 01$ & Manual ou Intelectual? \\
\hline$\# 02$ & Onde estão as fontes? \\
\hline$\# 03$ & Natural ou Social? \\
\hline$\# 04$ & Não Confunda! 01 \\
\hline$\# 05$ & O que é... Ciência? \\
\hline$\# 06$ & A crise de 29 \\
\hline$\# 07$ & Globalização \\
\hline$\# 08$ & Matemática \\
\hline$\# 09$ & O que é Materialismo Histórico Dialético? \\
\hline$\# 10$ & Fala Docente! \\
\hline$\# 11$ & Percurso Histórico Estrutural da EP Brasileira \\
\hline$\# 12$ & Final de Temporada \\
\hline$\# 13$ & A Cientificidade da Música \\
\hline$\# 14$ & Educação Profissional e Propedêutica \\
\hline$\# 15$ & Redação \\
\hline$\# 16$ & Cursos Técnicos \\
\hline$\# 17$ & Novas Exigências e Velhas Transformações \\
\hline$\# 18$ & Formação de Professores para EPT \\
\hline
\end{tabular}

Hasta el audio \#16 Cursos Técnicos, el tiempo de cada producción era de 15 minutos. A partir de esto, por lo tanto, \#17 nuevos requisitos y viejas transformaciones; y \#18 Formación docente para el EPT, fueron producidos y publicados con un tiempo total de 12 minutos. Este cambio fue promovido por las sugerencias de la audiencia. 


\section{CAPTURAR}

Tras la conclusión del esquema narrativo y la elaboración del primer guión, comenzó la producción de audio. Para ello, la captura se realizó a través del software Audacity, versión 2.3.1 (https://audacityteam.org) instalado en un microordenador portátil modelo Lenovo IdeaPad 310 y con la ayuda de un micrófono de condensador genérico, el BM-800 (Figura 2).

Figura 2 - Captura de audio.

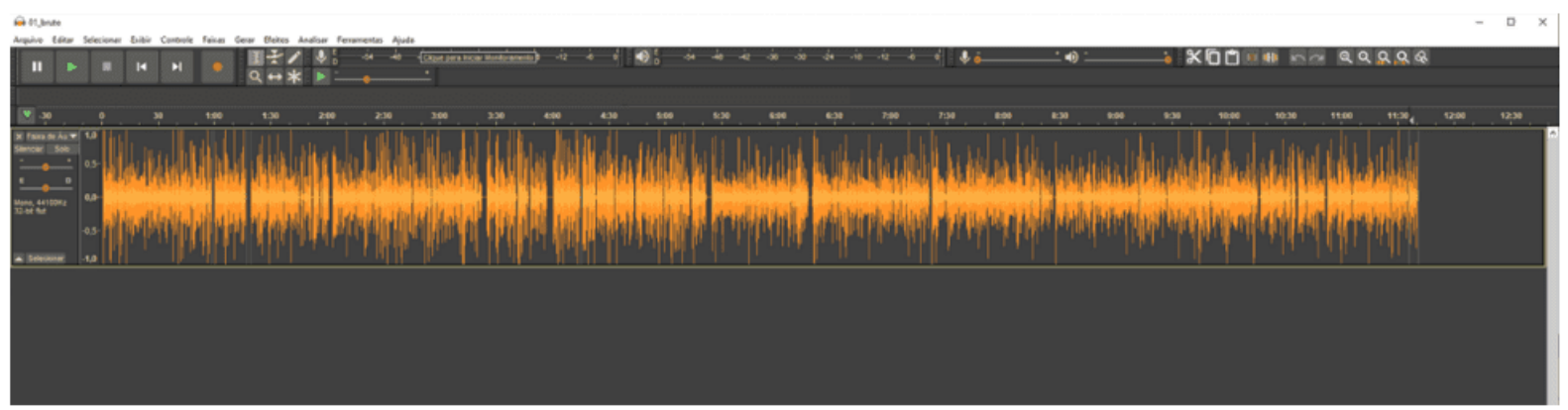

La captura de audio representó el inicio del proceso de materialización del objetivo previsto. Debido a la falta de experiencia en lo que se refiere a la narración de contenido escrito para la distribución de audio, el tamaño/volumen de la grabación era a veces más del doble del tiempo estipulado para cada audio. La figura anterior muestra el contenido grabado sin los errores del proceso de captura.

\section{EDICIÓN}

Después de capturar el audio y eliminar su excedente, se inició el proceso de edición. Para esta etapa, se utilizaron los recursos de audio de las plataformas YouTube (audiolibrary) (https://youtube.com/audiolibrary) para la inserción de pistas de música, y Freesound.org (https://freesound.org) para la inserción de efectos de sonido. Ambas plataformas cuentan con contenido sin costos financieros (Figura 3). 
Figura 3 - Edición de audio.

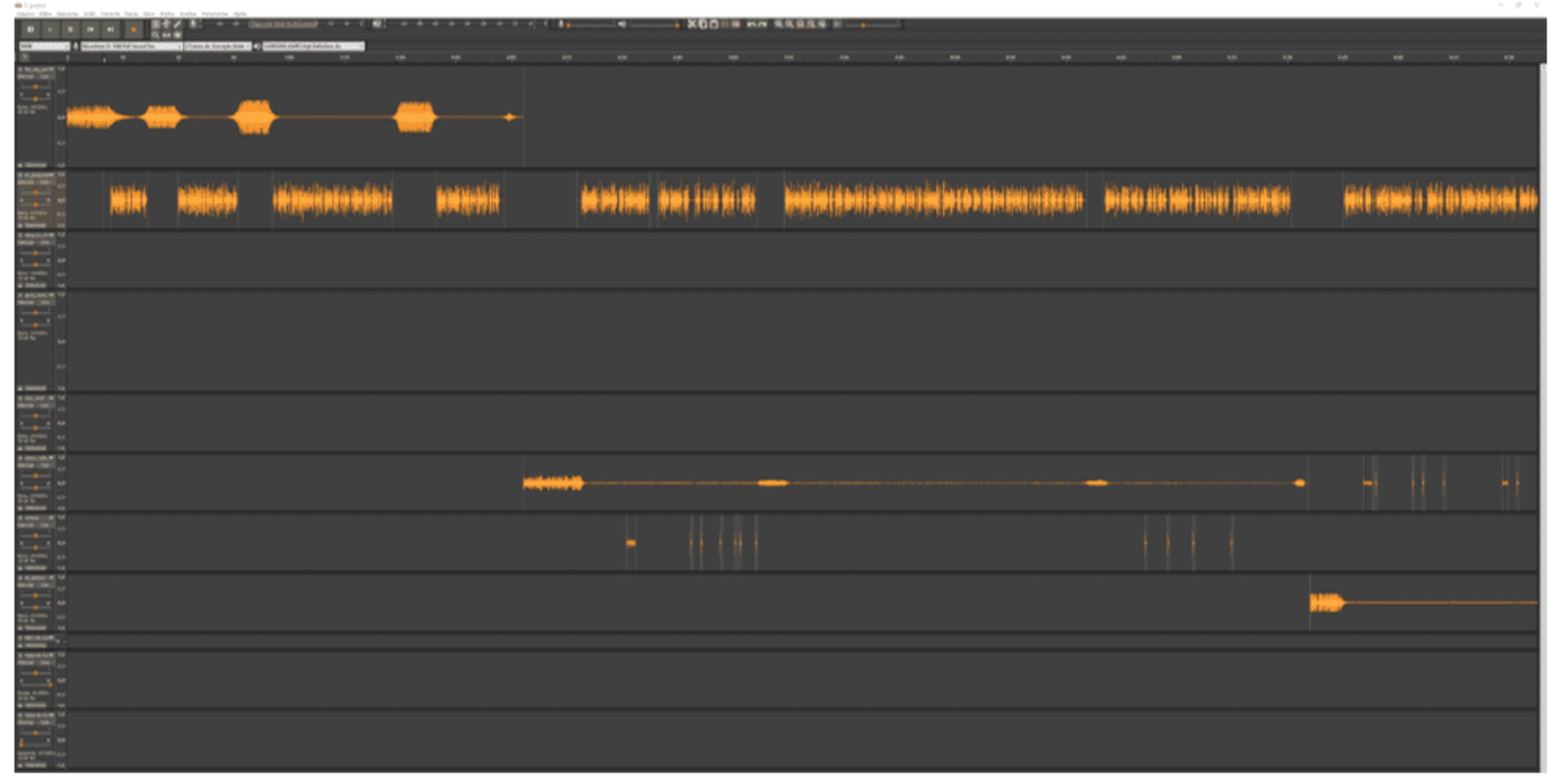

La opción de insertar pistas de música y efectos de sonido fue el resultado de la experiencia de consumo previo de contenidos distribuidos vía podcast. A lo largo del proceso de edición, también se percibió que este recurso, en algún nivel, se presentaba como capaz de suavizar la presencia de ciertas inserciones no deseadas, provenientes de la captura de audio en el entorno doméstico.

Con el fin de diferenciar el discurso del narrador con los argumentos originales de las fuentes de investigación, el contenido contó con la participación rotativa de 04 estudiantes de la Escuela Secundaria Integrada del Instituto Federal de Educación, siendo 02 de Amapá, campus Santana; y 02 de Rio Grande do Sul, campus Osório. En este sentido, los alumnos invitados se encargaron de narrar las citas directas insertadas en el texto del guión.

\section{DISTRIBUCIÓN}

Entre el gran número de opciones para la distribución de EPTCast, optamos por el Anchor.fm - http://anchor.fm. Esta fue una elección basada en dos fundamentos, a 
saber: Gratuidad total en su manejo; y Distribución automática de contenidos publicados en otras plataformas.

\section{IDENTIFICACIÓN DEL MATERIAL}

Al enviar el contenido producido a través del recurso dedicado a esta tarea (Click to upload or drag files here),se cargó el audio, lo que permitió la reproducción para que se pudiera comprobar la calidad del contenido a distribuir (Figura 4).

Figura 4 - Inserción del audio y la identificación del material.

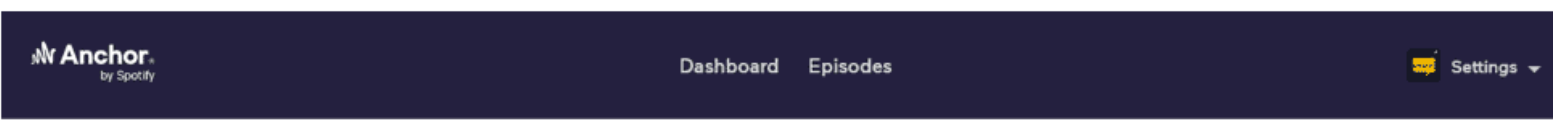

Create your episode

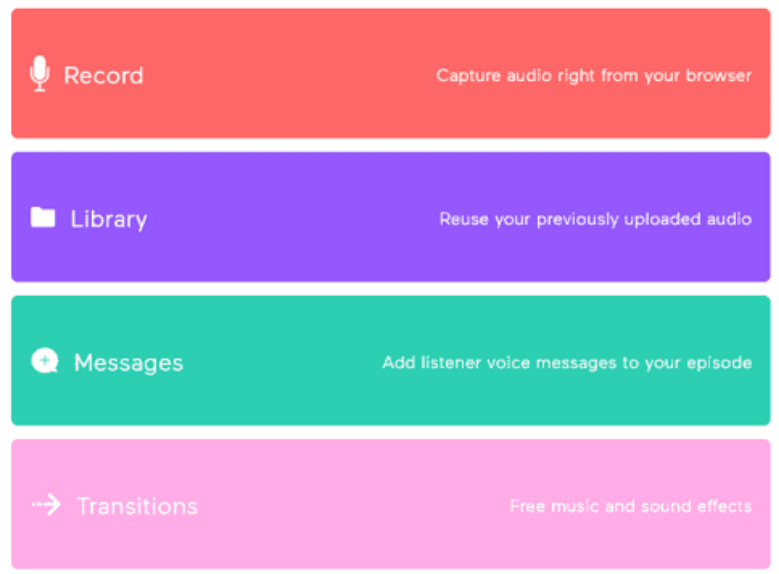

Save episode

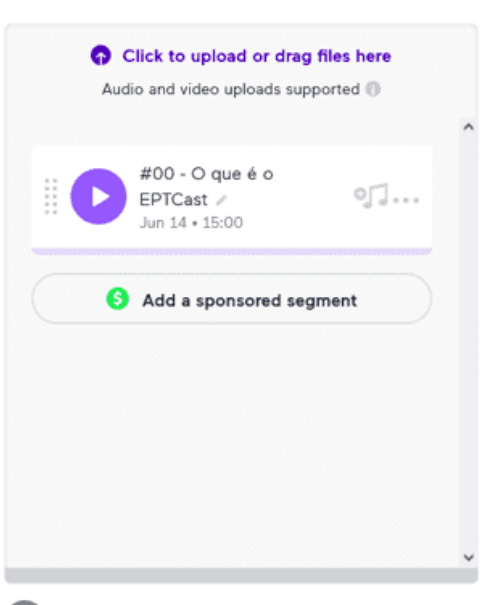

Total Length: $15 \mathrm{~m}$ 0s

Luego de insertar el audio, la plataforma solicitó información básica para identificar el contenido. Estos fueron: título (Episode title), descripción (Episode description), temporada (Season number), número de contenido (Episode number), tipo de contenido entre: completo, tráiler o bonificación (Full; Trailer; Bonus) y finalmente, si contenido fácil de usar o no (Clean; Explicit) (Figura 5). 
Figura 5 - Identificación del material.

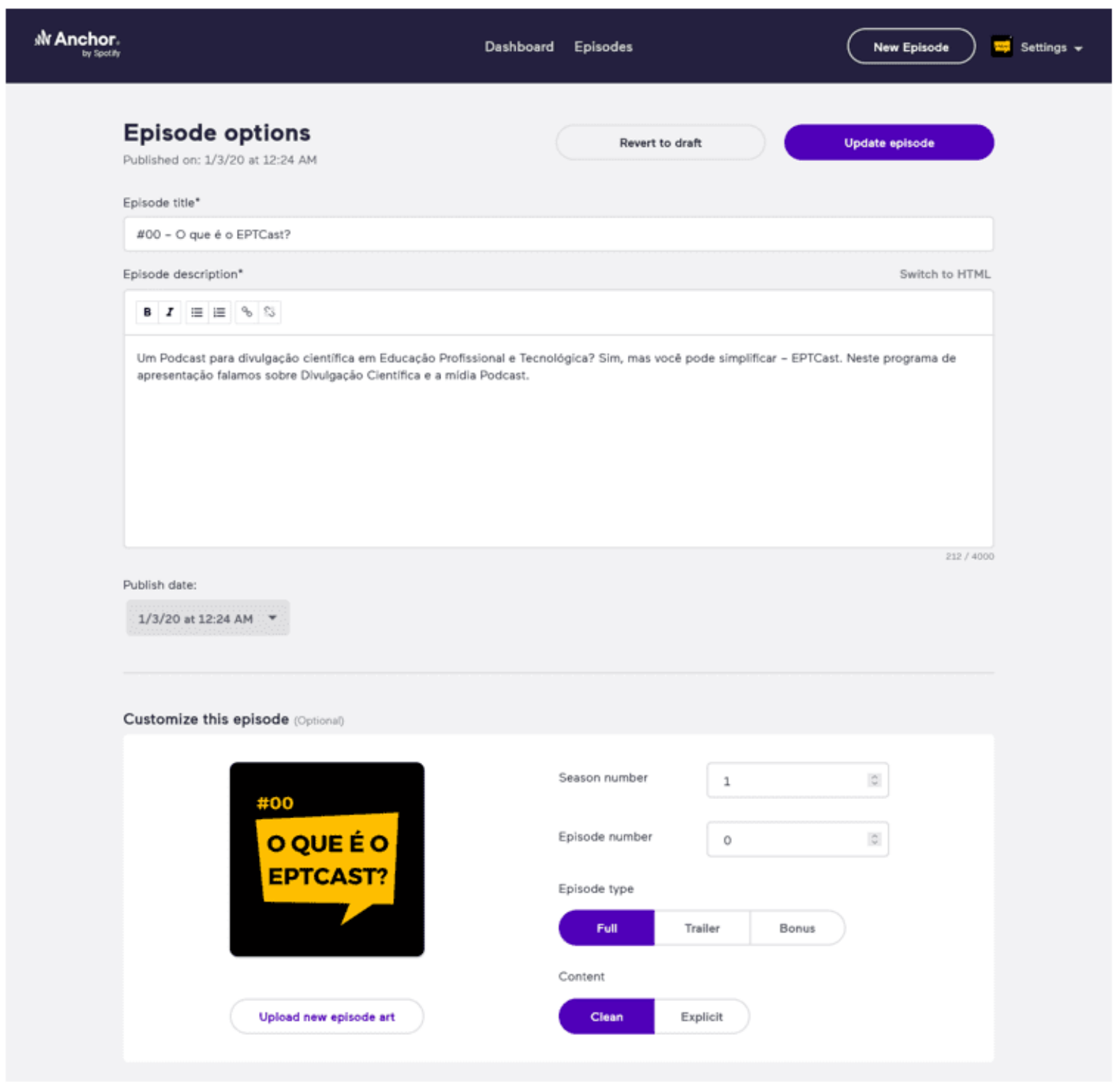

Tras la inserción de la información solicitada para la identificación del contenido, el audio se puso a disposición en esta plataforma, y también en la plataforma de servicios de streaming de música Spotify (https://spotify.com), permitiendo así su consumo en los primeros momentos. 


\section{PLATAFORMAS DE AUDIO DIGITAL}

A pesar de la automatización con respecto al proceso de distribución en otras plataformas, Anchor.fm no realizó esta tarea al mismo tiempo en todas las plataformas a las que está asociado. Sin embargo, a medida que el envío de audio aumentaba el contenido en online de EPTCast, se notó que las opciones de acceso estaban creciendo. En la publicación del \#07 globalización, se observó que la distribución alcanzó su número máximo de plataformas, 09 (Tabla 3).

Tabla 3 - Plataformas de acceso.

\begin{tabular}{c|c}
\hline NOME & ENDEREÇO (URL) \\
\hline Anchor & anchor.fm/eptcast \\
\hline Breaker & breaker.audio/eptcast \\
\hline Castbox & castbox.fm/channel/id2581365 \\
\hline Google Podcasts & google.com/podcasts?feed=aHROcHM6Ly9hbmNob3luZm0vc \\
\hline Apple Podcasts & podcasts.apple.com/us/podcast/eptcast/id1494934631 \\
\hline Overcast & overcast.fm/itunes1494934631/eptcast \\
\hline Pocket Casts & pca.st/8znyqbx5 \\
\hline RadioPublic & radiopublic.com/eptcast-WkaRNN \\
\hline Spotify & open.spotify.com/show/1hZ3KSkDDzOHuLmIPD6dh8
\end{tabular}

La distribución a través de la plataforma seleccionada se presentó como una experiencia de fácil comprensión y también de manejo simple. La inserción en otras plataformas digitales, de forma gratuita, se presentó como un importante diferencial ya que esta actividad demostró ahorrar tiempo, recurso aplicado en la realización de otras tareas de este Producto Educativo.

\section{CONSIDERACIONES FINALES}

Popularizar la cienticidad de la Educación Profesional y Tecnológica brasileña es lo que se pretendió a través de este trabajo - que no será el único. Por lo tanto, a través 
de lo expuesto en los apartados anteriores, se pretende contribuir a la popularización de una cienticidad que a veces aún no se entiende como tal.

El desarrollo social de las clases trabajadoras populares, a través del desarrollo de una cultura científica, es nuestra intención. En este sentido, hacemos uso de un medio descentralizado para popularizar estos temas que, a pesar de estar enfocados en la emancipación de quienes necesitan, hasta ahora, se encuentran y escuchan solo dentro del ciclo reservado.

A partir de aquí, nos encontramos esperanzados en mayores y mejores iniciativas encaminadas a popularizar estos temas.

\section{REFERENCIAS}

MOIRAND, S. et ali. La vulgarisation scientifique au croisement de nouvelles sphères d'activité langagière. Bakhtiniana, Rev. Estud. Discurso, vol. 11 n. 2, p. 137-161, 2016.

DE-LARA-GONZÁLEZ, A.; DEL-CAMPO-CAÑIZARES, E. El podcast como medio de divulgación científica y su capacidad para conectar con la audiencia. Revista Mediterránea de Comunicación, vol. 09 n. 1 p. 347-359, 2018.

RIVERA SALAS, P.E.; MONTOYA MÁRQUEZ, O. R. Evaluación de los podcasts de noticias: un acercamiento a emisoras de la ciudad de puebla. Revista de Comunicación de la SEECI. n. 48, p. 109-123, 2019.

DANTAS-QUEIROZ, M. V.; WENTZEL, L. C. P.; QUEIROZ, L. L. Science communication podcasting in Brazil: the potential and challenges depicted by two podcasts. An. Acad. Bras. Cienc., v. 90, n. 2, p. 1891-1901, 2018.

MOURA, D. H. Ensino médio integrado: subsunção aos interesses do capital ou travessia para a formação humana integral? Educ. Pesqui., v. 39, n. 3, p. 705-720, 2013. 
NDERITU PATRICK G. Bilingual naming of business premises, products and services in Murang'a county. Relatório de Projeto de Pesquisa (Mestre em Linguística) Universidade de Nairóbi, 2016.

LIU, X. Application of the Internet Technology in the Visual Communication Design. In: Atiquzzaman M., Yen N., Xu Z. (eds) BDCPS 2019, AISC 1117, p. 1701-1706, 2020.

MILLER, D. W.; TOMAN, M. An analysis of rhetorical figures and other linguistic devices in corporation brand slogans. Journal of Marketing Communications, v. 22, n. 5, p. 474-493, 2014.

Enviado: Junio de 2021.

Aprobado: Junio de 2021. 